\title{
OBSERVATIONS OF FIXED AND MOBILE CHARGE IN COMPOSITE MEMS RESONATORS
}

\author{
G. Bahl ${ }^{1}$, R. Melamud ${ }^{1}$, B. Kim ${ }^{13}$, S. Chandorkar ${ }^{1}$, J. Salvia ${ }^{1}$, M.A. Hopcroft ${ }^{13}$, R.G. Hennessy ${ }^{1}$, \\ S. Yoneoka ${ }^{l}$, C.M. Jha ${ }^{l}$, G. Yama ${ }^{2}$, D. Elata ${ }^{l}$, R.N. Candler ${ }^{2}$, R.T. Howe ${ }^{1}$, and T.W. Kenny ${ }^{l}$ \\ ${ }^{1}$ Stanford University, Stanford, CA, USA $\quad{ }^{2}$ Robert Bosch Corporation, USA \\ ${ }^{3}$ currently at University of California Berkeley, CA, USA
}

\begin{abstract}
This paper investigates dielectric charge in oxide-coated composite-beam silicon electrostatic resonators. The effects of charge in the oxide layers on resonant frequency are theoretically modeled. Evidence for the presence of both fixed and mobile charge is presented through experimental studies. It is shown that the motion of charge is controllable through the bias voltage applied to the resonator. These studies are relevant for improving the reliability of non-contacting MEMS where dielectrics may be present such as timing references, inertial sensors, and optical actuators.
\end{abstract}

\section{INTRODUCTION}

Dielectric charging in contacting structures such as RF capacitive switches has been studied extensively. In these structures capacitance measurements are used to infer charge through partial deflection or complete pull-in measurements [1, 2]. In stiff non-contacting structures such as resonators, accelerometers and gyroscopes, charge build-up can also affect the stability and reliability of the transducer. Pull-in measurements are often impractical for such structures and do not emulate standard operating conditions. Here, the use of resonant frequency measurements can complement pull-in measurements as a noncontact method to study the evolution of charge in dielectrics under low-field conditions.

We utilize oxide-coated composite-beam silicon resonators designed for passive temperature compensation as test structures [3]. This paper presents evidence for trapped charge in the resonator oxides by two methods: observing frequency dependence on bias voltage to show the existence of built-in electric fields within the resonating device, and examination of transient behavior of frequency under controlled bias and temperature conditions.

\section{DEVICE DESCRIPTION}

\section{Resonator fabrication}

Single-anchor double-ended tuning fork resonators are fabricated on $20 \mu \mathrm{m}$ device layer SOI wafers using the "epi-seal" single-wafer encapsulation process (Fig.1) [4]. Each resonator beam is $200 \mu \mathrm{m}$ in length and the width $W$ is the design variable.

The devices fabricated in this technology are vacuum encapsulated with pristine silicon surfaces and are protected from environmental agents. Native oxide on silicon surfaces is destroyed during the high temperature sealing step performed during the final steps of the encapsulation process. No additional oxide is expected to grow within the encapsulation since nitrogen and hydrogen are flooded in during the sealing step and are later annealed out. Longterm frequency stability within the encapsulation has previously been shown for silicon-only devices [5].

\section{Dielectric Coating}

An oxidation step can be performed prior to sealing the devices with the silicon cap layer. The oxide is thermally grown at $1100^{\circ} \mathrm{C}$ in a wet ambient. The process duration varies with the oxide thickness desired. Since this oxide is significantly thicker (compared to a native oxide layer) it can survive the high temperature sealing step.

The SOI wafers used in fabrication have a (100) crystal

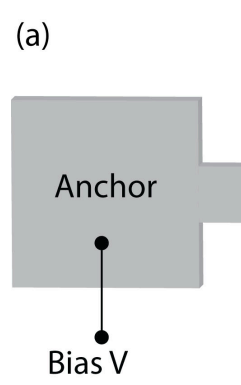

(b)
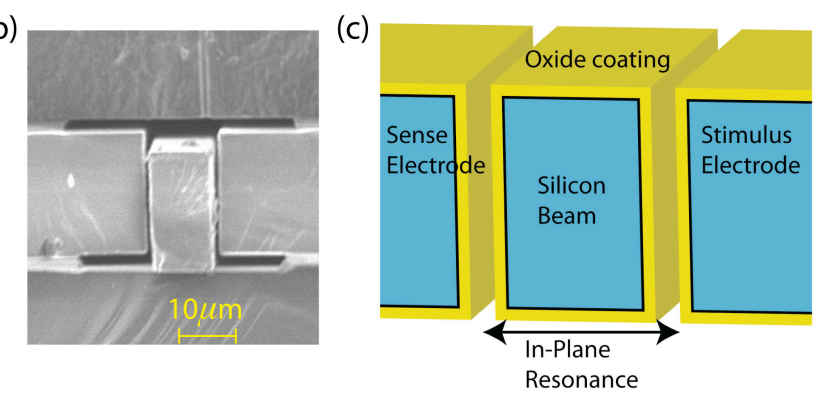

Figure 1: (a) Top view schematic of resonator with electrical setup of the device. (b) SEM of cross-section shown. (c) Schematic of SEM cross-section.

orientation at the surface. The devices are oriented such that the etched device layer side-walls primarily expose the (110) plane. The oxide thickness on the wafer surface is measured and the corresponding sidewall oxide thickness can then be estimated using the Deal-Grove oxidation model [6]. The devices presented in this paper have an estimated $0.45 \mu$ m oxide coating on (110) surfaces.

\section{Electrical setup}

In all experiments the resonator is connected to a source of fixed bias voltage $V$ (Fig.1). The stimulus and sense electrodes are nominally held at ground, though they do also support a small amplitude AC resonance signal. All AC signals are assumed to be negligible in the theoretical analysis, though higher order effects are expected for large signal amplitudes [7].

\section{MODELING CHARGE EFFECTS}

Each resonator beam forms two capacitors with the stimulus and sense electrodes adjoining it. The resonator beam height defined by the device layer $(\sim 20 \mu \mathrm{m})$ and length $(L \sim 200 \mu \mathrm{m})$ are significantly greater than the gap width $(\sim 1.5 \mu \mathrm{m})$ so it is reasonable to model these capacitors with the parallel plate approximation. The stimulus and sense electrodes are nominally held at DC ground potential. Thus, the four individual capacitors for both tuning fork beams are structurally and electrically (DC) identical and can thus be treated as a single capacitor with the same bias voltage across it but with four times the area (Fig.2). The spatial distribution of charge within the dielectrics is unknown but can be modeled as an equivalent uniform surface charge at the vacuum-dielectric interface.

Electrostatic spring softening explains how the resonance frequency of a parallel-plate resonator depends on the applied bias 


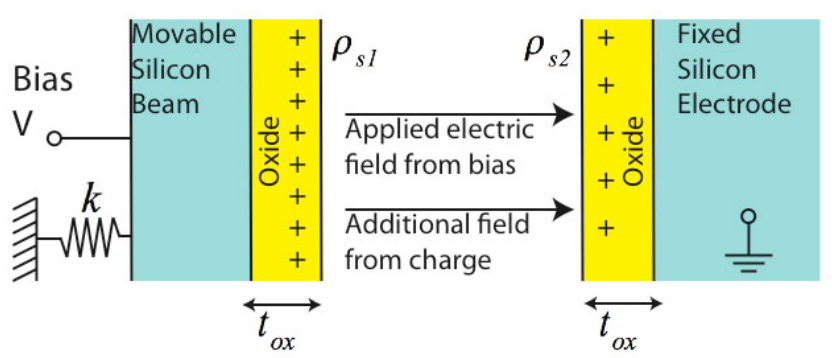

Figure 2: Single-capacitor equivalent model of the resonator system.

voltage [8]. In the absence of additional electric fields, the polarity of the bias voltage does not affect the spring softening since the voltage term is squared. However, when charge is present in the dielectrics additional field may arise within the actuation gap. This additional field can be modeled as a built-in voltage $\Delta V$, additive or subtractive to the applied bias depending on bias polarity. This changes the effective bias voltage and modifies the frequency.

Under electrical spring softening with small resonance amplitude the resonant frequency is calculated as

$$
f \approx f_{o}\left(1-\frac{\Delta k_{e}}{2 k}\right)
$$

where $k$ is the mechanical spring constant. The electrical spring softening $\Delta k_{e}$ due to both charge and applied bias voltage can be analytically calculated. Specifically, the dependence on voltage can be calculated to be

$$
\Delta k_{e} \propto(V+\Delta V)^{2}
$$

The built-in voltage can be expressed as a function of the uniform vacuum-oxide surface charge densities as follows

$$
\Delta V=\frac{t_{o x}}{\epsilon_{o x}}\left(\rho_{s 1}-\rho_{s 2}\right)
$$

If the amount of surface charge on the two dielectrics is identical i.e. $\rho_{s 1}=\rho_{s 2}$, the built-in electric field will be canceled out and there is no effect on frequency.

Using equations 1-3, when frequency is plotted as a function of bias voltage $\Delta V$ appears as a translation along the voltage axis. As a consequence when frequency is plotted against magnitude of bias, the frequency appears to shift in opposite directions depending on bias polarity (see Fig.3). Although the zero charge state $(\Delta V=0)$ cannot be observed in a given resonator that has charge built-in, the polarity dependent splitting of the frequency-

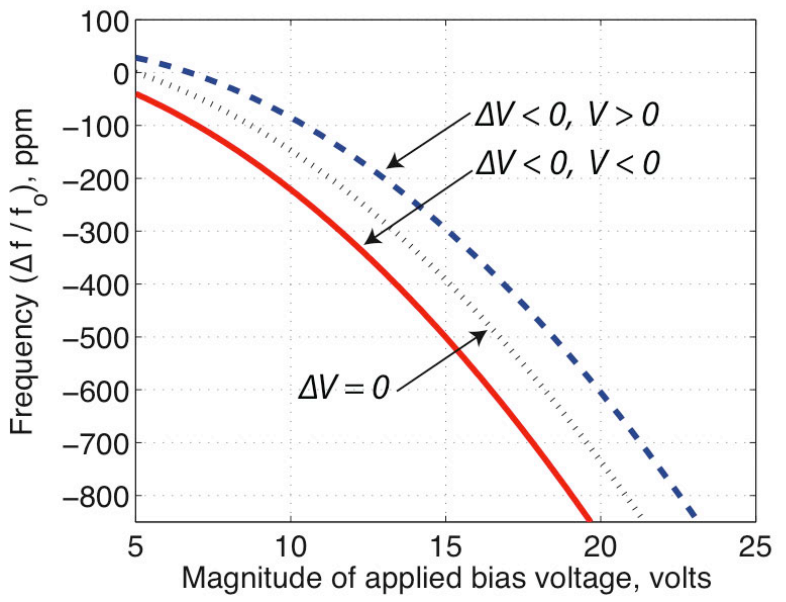

Figure 3: Simulation result for a $1 M H z$ resonator based on the charge model with the $\Delta V=0$ and $\Delta V<0$ cases plotted. Resonance frequency depends on bias polarity when charge is present in the oxide coating, i.e. when $\Delta V \neq 0$.

bias curve should still be seen. In recently published work the use of frequency-bias curves to extract the uncharged mechanical resonance frequency has been discussed [9].

\section{RESULTS}

Environmental factors (such as humidity) known to affect charge states on dielectrics cannot act on the sealed devices. The primary control variables on the system are temperature and bias voltage. The possibility of unintentional electrostatic discharge occurring within the encapsulation and having affected the behavior of these devices is unlikely, though it cannot be entirely ruled out.

\section{Fixed oxide charge}

The frequency of the resonant peak is monitored for various bias voltages and is plotted against the magnitude of bias. All measurements were made in a temperature-stabilized oven using a HP 4395A Network Analyzer with an amplifier at the resonator output to boost the signal. By fitting this data to the theoretical model $\Delta V$ can be extracted. Some representative data sets are shown in Fig.4. (a) Experiment : Oxide-coated resonator

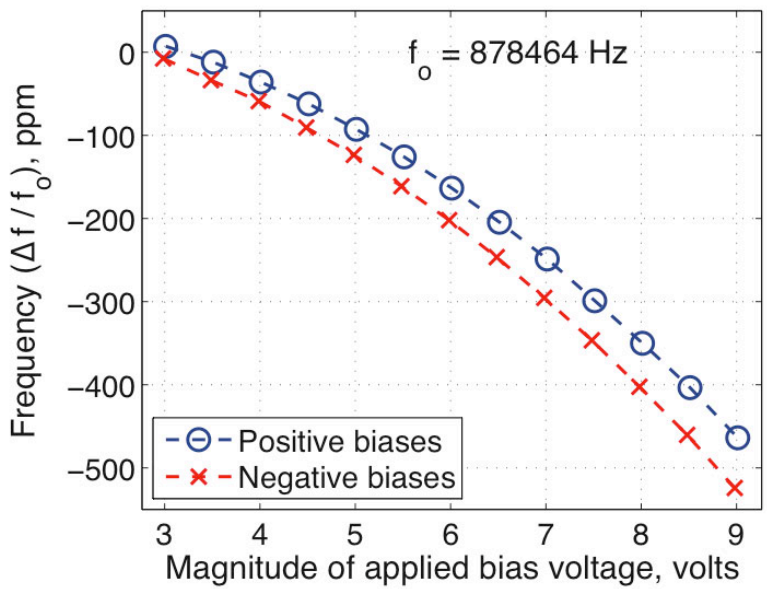

(b) Experiment : Oxide-free silicon resonator

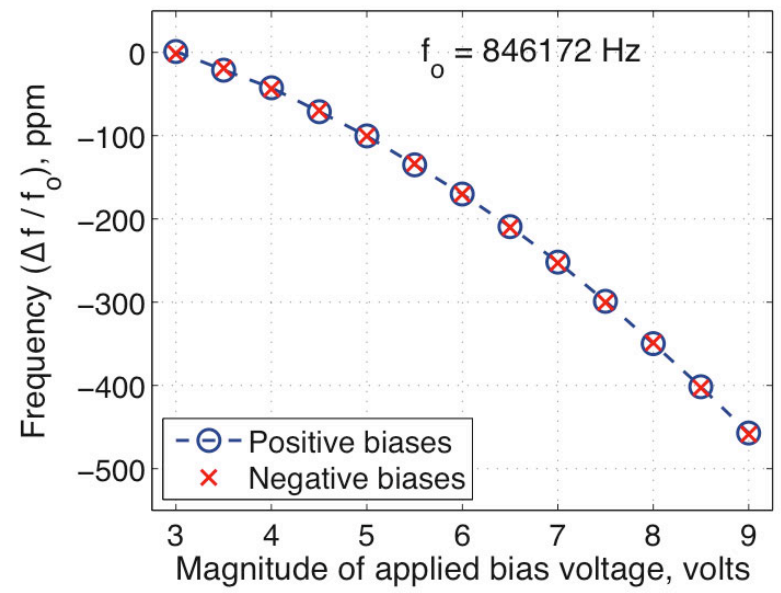

Figure 4: Bias polarity dependence in oxide-coated resonators. (a) Oxide-coated resonators show both positive and negative $\Delta V$ indicating the presence of fixed charge in the dielectric. The frequency-bias curve of a resonator with $\Delta V<0$ is shown here. (b) Oxide-free silicon devices show no measurable bias polarity dependence. 
(a) Fast transients observed in some oxide coated resonators

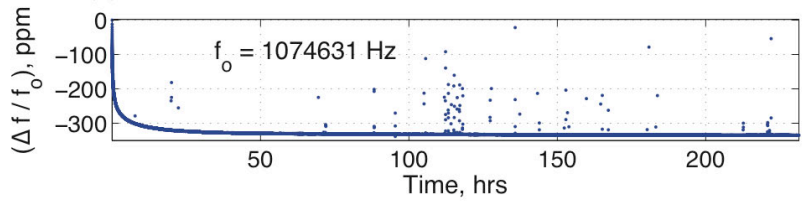

(b) Slow drift behavior observed in some oxide coated resonators

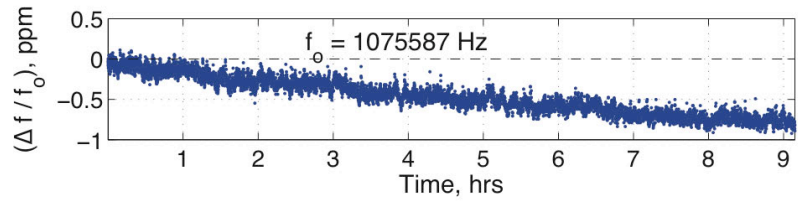

Figure 5: Frequency drift behavior seen in some oxide-coated resonators.

Of the 21 oxide-coated resonators that were tested all exhibit non-zero $\Delta V$ values in the range of $-250 \mathrm{mV}$ to $+340 \mathrm{mV}$. Notably, most oxide-coated resonators from this wafer exhibit negative values of $\Delta V$. In these resonators we note that an observed voltage shift of $100 \mathrm{mV}$ corresponds to an equivalent surface charge imbalance $\left(\rho_{s 1}-\rho_{s 2}\right)$ of approximately $7.67 \times 10^{-10} \mathrm{C} / \mathrm{cm}^{2}$ as calculated from equation 3 . The reasons for this variation in oxide charge imbalance over the wafer and predominance of negative values of $\Delta V$ are being investigated. Silicon-only resonators of identical design were also tested ( 9 devices) and showed no measurable frequency asymmetry (fitted $\Delta V=-1 \pm 4 \mathrm{mV}$ ).

\section{Mobile oxide charge}

Mobile charge observations are made with the oxide-coated resonators constantly actuated at their resonant frequency when connected in a closed-loop oscillator circuit. Frequency readings are taken using an Agilent 53132A Universal Frequency Counter.

Some devices exhibit large decaying transients over short durations immediately after the bias voltage is changed. These transients are the focus of this study (Fig.5a). Additionally, some devices show very little drift over long durations of time, and no transients (Fig.5b). Finally, many devices appear to show no drift or transient behavior over currently tested observation intervals and are usable for frequency stability experiments. The reason why certain oxide-coated resonators exhibit frequency transients while others do not is presently under investigation. This behavior is absent in silicon-only resonators made with the same "epi-seal"

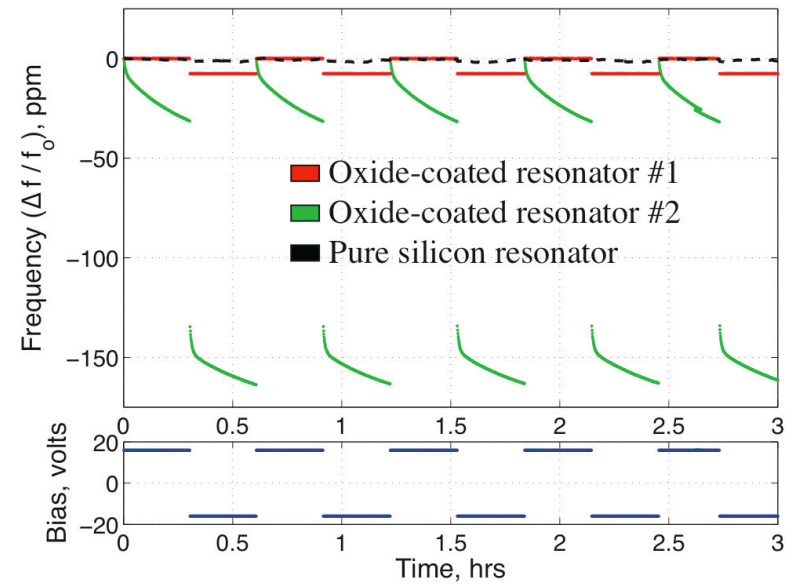

Figure 6: Frequency trends of three lithographically identical devices $(W=5.75 \mu \mathrm{m})$ showing transients and bias polarity dependence. technology [5].

The bias-frequency sensitivity of these resonators can be extracted from the frequency-bias curves shown previously and is around 60-100 ppm/Volt depending on beam width $W$. Bias voltage is controlled using a Valhalla $2701 \mathrm{C}$ voltage calibrator to a stability better than $1 \mathrm{mV}$. As a result the bias voltage cannot account for the observed transients in frequency. Although temperature is kept constant in a stabilized oven environment there are still some random fluctuations that affect the frequency. Since the resonators are passively temperature-compensated with the oxide [3], temperature fluctuations can only account for frequency variations on the order of $3 \mathrm{ppm}$ or less at the operating temperature of $40^{\circ} \mathrm{C}$. The oscillator circuit boards are reusable and are often exchanged between devices to ensure that any drifts are not oscillator artifacts.

Fig.6 shows the frequency trends of three lithographically identical devices $(W=5.75 \mu \mathrm{m})$ as the bias is alternated between $+18 \mathrm{~V}$ and $-18 \mathrm{~V}$. Bias polarity dependence of frequency is visible in the oxide-coated resonators. In addition, oxide-coated resonator \#2 shows transient behavior in time. Both effects are absent in silicon resonators and the data from one silicon device is shown. Reproducibility of the short duration transients in oxide-coated resonators strongly indicates that shape change, fatigue, and other burn-in processes or material issues can be ruled out.

The transient behavior of frequency can be controlled using various bias alternation schemes. In the experiment shown in Fig.7, a negative bias $V_{2}$ is applied momentarily to partially reverse the effect of charge movement due to positive bias $V_{l}$. Frequency readings are not taken at bias $V_{2}$ since the oscillator does not operate at low bias magnitudes $\left(V_{2}=0 \mathrm{~V},-5 \mathrm{~V}\right.$ in this experiment). A comparison of four experiments plotted against a common reference frequency $f_{o}$ reveals a level of control over the net frequency drift.

Since the frequency is seen to be a function of the biasing history, to replicate an experiment the initial conditions and stimuli must be restored exactly. The dielectrics have an unknown volume charge distribution, however, and the only observable quantities from the system are the frequency and amplitude of resonance. It is thus unlikely that the actual charge distribution at the beginning of any experiment can be determined or restored using this measurement method.

Transients in frequency at a fixed bias voltage seem to

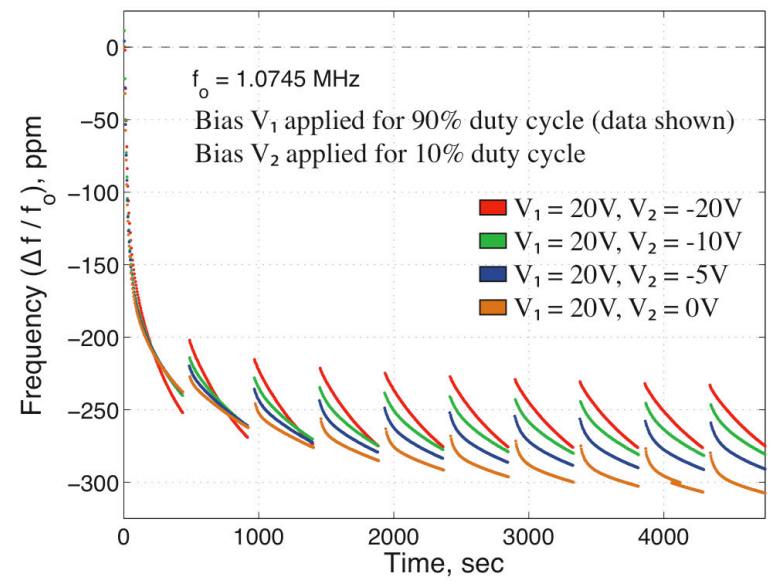

Figure 7: Bias control of frequency transients in an oxide-coated resonator. Multiple experiments are superimposed to show frequency dependence on biasing scheme. 


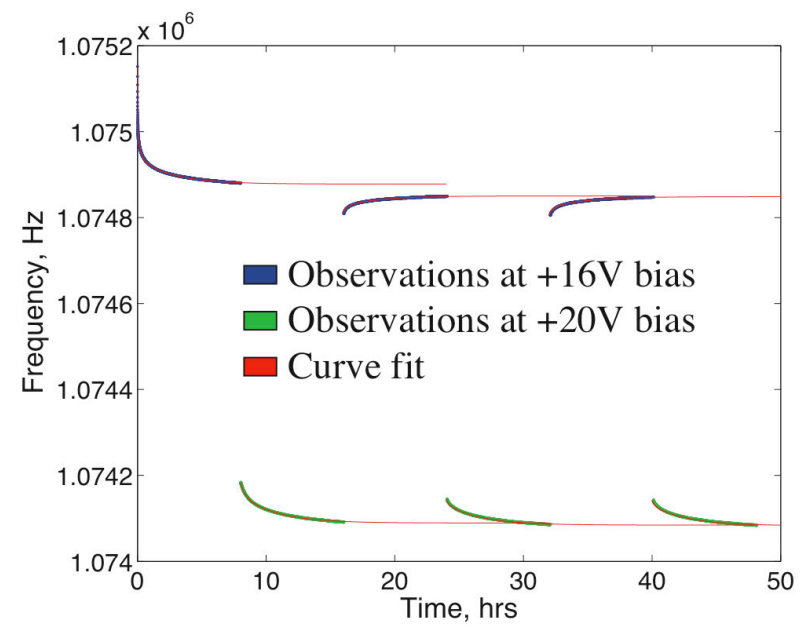

Figure 8: Demonstration of bias-dependent steady states.

approach a steady state when given enough time to stabilize. As is seen in Fig.7, however, the transients reappear at the same voltage $V_{l}$ after the devices are rested for some time with zero applied voltage. This suggests that there may be a restoring effect acting on the charge that does not require an externally applied electric field.

This effect can be further studied with the experiment shown in Fig.8. Here, the bias voltage polarity is maintained constant at all times while its magnitude is varied. The device is initially primed by bringing the frequency close to its steady state at $V=16 \mathrm{~V}$ bias. This transient can be interpreted as an increase of $\Delta V$ with time under the influence of the applied electric field (charge redistribution) to reach an equilibrium state. The bias voltage $V$ is then increased to $20 \mathrm{~V}$ to increase the electric field across the gap. The frequency is allowed to drift to its new steady state. This second downward transient indicates a further movement of charge and a further increase in $\Delta V$. Then when the field is reduced by reducing applied bias to $16 \mathrm{~V}$, the frequency is observed moving in the opposite direction back towards the $16 \mathrm{~V}$ state. This indicates that $\Delta V$ is decreasing over time although the applied electric field is still in the same direction (since bias polarity is maintained). It must be concluded then that the applied electric field is no longer the dominant driving force on the charge. It is seen that this back and forth movement between two steady states happens consistently as bias is alternated in this manner.

It should also be noted that frequency transients appear to accelerate with increasing temperature, though the specific time constants and temperature-dependence is a direction for future study. It is yet to be determined whether the long-duration drifts are also caused by mobile charge.

\section{CONCLUSIONS}

The devices that show frequency transients offer an excellent opportunity to study the behavior, mechanisms and mitigation of mobile charge. It must be noted that there are also oxide-coated resonators fabricated in this same process and on the same wafers that exhibited much smaller bias splits and much smaller transients and smaller long-term drifts. The presence of a significant fraction of oxide-coated resonators that do not exhibit the effects described in this paper is an important indication that the elimination of these effects may be possible.
Currently we are investigating process non-uniformities, plasma charging, and other possible sources of the trapped charge, and the negative predominance of the built-in potential $\Delta V$ in nontransient devices. Hermeticity of the resonator environment and the ability to control the amount of oxide in identical resonators provides a well-controlled and highly sensitive scientific tool to non-destructively study the effects and time-evolution of charge on dielectrics in MEMS under low-field conditions.

\section{REFERENCES}

[1] C. Goldsmith, J. Ehmke, A. Malczewski, B. Pillans, S. Eshelman, Z. Yao, J. Brank, and M. Eberly, "Lifetime Characterization of Capacitive RF MEMS Switches," IEEE International Microwave Symposium, 1, (2001)

[2] R.W. Herfst, H.G.A. Huizing, P.G. Steeneken, and J. Schmitz, "Characterization of Dielectric Charging in RF MEMS Capacitive Switches," IEEE International Conference on Microelectronic Test Structures, (2006), pp.133-136

[3] R. Melamud, B. Kim, S.A. Chandorkar, M.A. Hopcroft, M. Agarwal, C.M. Jha, and T.W. Kenny, "TemperatureCompensated High-Stability Silicon Resonators," Applied Physics Letters, 90, 244107

[4] R.N. Candler, M.A. Hopcroft, B. Kim, W.-T. Park, R. Melamud, M. Agarwal, G. Yama, A. Partridge, M. Lutz, and T.W. Kenny, "Long-Term and Accelerated Life Testing of a Novel Single-Wafer Vacuum Encapsulation for MEMS Resonators," Journal of Microelectromechanical Systems, 15, (2006), pp.1446-1456

[5] B. Kim, R.N. Candler, M.A. Hopcroft, M. Agarwal, W.-T. Park, and T.W. Kenny, "Frequency Stability of Wafer-Scale Film Encapsulated Silicon Based MEMS Resonators," Sensors and Actuators, A: Physical, 136, pp.125-131, (2007)

[6] J.D. Plummer, M.D. Deal, and P.B. Griffin, Silicon VLSI Technology. New Jersey: Prentice Hall, 2000

[7] M. Agarwal, S.A. Chandorkar, R.N. Candler, B. Kim, M.A. Hopcroft, R. Melamud, C.M. Jha, T.W. Kenny, and B. Murmann, "Optimal Drive Condition for Nonlinearity Reduction in Electrostatic Microresonators," Applied Physics Letters, 89, 214105, (2006)

[8] S.G. Adams, F.M. Bertsch, K.A. Shaw, P.G. Hartwell, F.C. Moon, and N.C. MacDonald, "Capacitance Based Tunable Resonators,” J. Micromech. MicroEng., 8, (1998), pp.15-23

[9] S. Kalicinski, H.A.C. Tilmans, M. Wevers, and I. De Wolf, "A New Method to Determine the Mechanical Resonance Frequency, Quality Factor and Charging in Electrostatically Actuated MEMS," IEEE MEMS 2008, Tuscon, Arizona, USA, Jan 13-17, (2008)

\section{ACKNOWLEDGEMENTS}

This work was supported by DARPA CIEMS, Bosch, Epson, HP, Agilent, Boeing, Qualcomm, DARPA HERMIT (ONR N66001-03-1-8942), and the National Nanofabrication Users Network facilities funded by the National Science Foundation under award ECS-9731294, and the National Science Foundation Instrumentation for Materials Research Program (DMR 9504099), and the Stanford Graduate Fellowship support for R. Melamud. We thank Andrew Graham, Wes Smith and Scott Lord for their support. 